\title{
A class of symmetric and non-symmetric band matrices via binomial coefficients
}

https://doi.org/10.1515/spma-2020-0142

Received October 16, 2020; accepted May 29, 2021

Abstract: Symmetric matrix classes of bandwidth $2 r+1$ was studied in 1972 through binomial coefficients. In this paper, non-symmetric matrix classes with the binomial coefficients are considered where $r+s+1$ is the bandwidth, $r$ is the lower bandwidth and $s$ is the upper bandwidth. Main results for inverse, determinants and norm-infinity of inverse are presented. The binomial coefficients are used for the derivation of results.

Keywords: Symmetric and Non-symmetric matrix, Binomial coefficients, Gaussian $q$-Binomial coefficients, generalized Fibonomial coefficients, Odd and Even banded Toeplitz matrices

\section{Introduction}

Toeplitz matrices are square matrices having constant entries along their diagonals. They occur in many research fields. These kinds of matrices can either be finite or infinite. Applications are found in Variational structure, Matrix theory, Theory of differential equations, Time series analysis, Signal and Image processing, Markov Chains, Queueing theory, etc. In Numerical Analysis, Toeplitz matrices are applied in Finite difference schemes, Finite element methods, Spline methods, Boundary value methods, Block unification methods, Partial differential equation and many more. Symmetric matrices have been studied by several researchers (see Refs. [2, 7, 16, 20, 25]).

Arikan and Kilic [1] defined the Toeplitz matrices as special types of square matrices in which the entries on each descending diagonal from left to right are constant. Herein, a Toeplitz matrix $A(r+s+1, n)$ represents an $n \times n$ band matrix of bandwidth $r+s+1$ where $r$ is the lower bandwidth and $s$ is the upper bandwidth.

In 1972, the authors [16], derived the formulae

$$
\begin{gathered}
\operatorname{det}(A(2 r+1, n))=(-1)^{n+r-1} \prod_{k=1}^{n}\left(\begin{array}{c}
k+2 r-1 \\
r
\end{array}\right)\left(\begin{array}{c}
k+r-1 \\
r
\end{array}\right)^{-1}, \\
\left\{A(r+s+1, n)^{-1}\right\}_{i, j}=(-1)^{r}\left(\begin{array}{c}
i+r-1 \\
r
\end{array}\right)\left(\begin{array}{c}
j+r-1 \\
r
\end{array}\right) \sum_{k=i}^{n} \frac{\left(\begin{array}{c}
k+r-1-i \\
r-1
\end{array}\right)\left(\begin{array}{c}
k+r-1-j \\
r-1
\end{array}\right)}{\left(\begin{array}{c}
k+r-1 \\
r
\end{array}\right)\left(\begin{array}{c}
k+2 r-1 \\
r
\end{array}\right)}
\end{gathered}
$$

and

$$
\left\|A(2 r+1, n)^{-1}\right\|_{\infty}=\left\{\begin{array}{l}
\frac{\prod_{k=1}^{r}(n+2 k-1)^{2}}{2^{2 r}(2 r) !} \text { for odd } \mathrm{n} \\
\frac{\prod_{k=1}^{r}(n+2 k-2)^{2}}{2^{2 r}(2 r) !}\left(\frac{n+2 r}{n}\right) \text { for even } \mathrm{n} .
\end{array}\right.
$$

\footnotetext{
*Corresponding Author: Omojola Micheal: Department of Mathematical Sciences, The Federal University of Technology, Akure, Nigeria, E-mail: omojolamts116762@futa.edu.ng, omojola.micheal@yahoo.com Emrah Kilic: TOBB University of Economics and Technology Department of Mathematics 06560, Ankara Turkey, E-mail: ekilic@etu.edu.tr
} 
Also, the authors gave the constructive approach for the inverse of the symmetric matrix $A(2 r+1, n)$ as

$$
\begin{aligned}
\left\{A(2 r+1, n)^{-1}\right\}_{i, j} & =-\frac{(n+1-i)(n+2-i) \cdots(n+r-i)}{(r-1) !(2 r-1) !} \\
& \times \sum_{k=0}^{r-1}\left[(-1)^{k}\left(\begin{array}{c}
r-1 \\
k
\end{array}\right) \frac{\prod_{r=0}^{r-1}(i+r)}{(i+k)} \frac{(j+k+r-1) !}{(j+k-r) !} \frac{(n+k) !}{(n+k+r) !}\right], \quad i \geq j, \quad r \geq 1 .
\end{aligned}
$$

Recently, Arikan and Kilic [1] studied the matrix

$$
a_{i j}=(-1)^{r+i-j}\left(\begin{array}{c}
r+s \\
r+i-j
\end{array}\right),
$$

where $s$ is the upper bandwidth and $r$ is the lower bandwidth. The following results were presented in Gaussian $q$-binomial, generalized Fibonomial and binomial forms.

$$
\begin{gathered}
\operatorname{det} C_{n}=i^{n r(r-1)} \prod_{m=0}^{n-1}\left\{\begin{array}{c}
r+s+m \\
r+m
\end{array}\right\}_{U}\left\{\begin{array}{c}
s+m \\
m
\end{array}\right\}_{U}^{-1}, \\
h_{k j}^{-1}=(-1)^{r(j+k+r-1)+j} i^{(k-j)(r-s+1)} q^{\frac{1}{2}\left((r-s)(j-k)+k^{2}+j\right)}\left[\begin{array}{c}
r+s+j \\
r
\end{array}\right]_{q}^{-1} \\
\times \sum_{k \leq d \leq j}(-1)^{d} q^{\frac{1}{2}\left(d^{2}-d\right)-k d}\left[\begin{array}{c}
s \\
d-k
\end{array}\right]_{q}\left[\begin{array}{c}
r+s+k \\
s-d+k
\end{array}\right]_{q}\left[\begin{array}{c}
s+k \\
d
\end{array}\right]_{q}^{-1}\left[\begin{array}{c}
j-d+r-1 \\
r-1
\end{array}\right]_{q}\left[\begin{array}{c}
r+d \\
r
\end{array}\right]_{q}
\end{gathered}
$$

and

$$
\left\|A^{-1}\right\|_{\infty}=\frac{(r+t+1)^{-}(s+n-t)^{\underline{n}}}{(r+s) !},
$$

where $t=\left\lfloor\frac{n r}{r+s}\right\rfloor$ and the falling factorial is defined as $x^{\underline{n}}=x(x-1) \cdots(x-n+1)$.

For more on Gaussian $q$-binomial, generalized Fibonomial and binomial forms (see Arikan and Kilic [1]). Results were presented for $0 \leq k, j \leq n-1$. Unfortunately, the constructive approach has not been studied since 1972. It is quite easy to run into trouble because one may not know if the above summand (5) (in its binomial form) is singular in the interval of summation. Hence, the need to use assumptions or parametric option.

On the other hand, the modeling of inverses of non-symmetric matrices in terms of dimension has been difficult. The author, Murray Dow [8], studied the matrix

$$
A(r+s+1, n)=\left[\begin{array}{ccccccc}
-3 & 1 & & & & \\
3 & -3 & 1 & & & \\
-1 & 3 & -3 & 1 & & \\
& -1 & 3 & -3 & 1 & \\
& & \ldots & \ldots & \ldots & \\
& & -1 & 3 & -3 & 1 \\
& & & -1 & 3 & -3
\end{array}\right],
$$

where $r=2, s=1$ and gave its determinant as

$$
\operatorname{det} A(4, n)=\frac{(n+1)(n+2)}{2}
$$

and its inverse is given as

$$
A_{i j}^{-1}= \begin{cases}a_{0}(j) i(i+1) & \text { for odd } \mathrm{n} \\ b_{0}(j) i^{2}+b_{1}(j) i+b_{2}(j) & \text { for even } \mathrm{n}\end{cases}
$$




$$
\begin{aligned}
& a_{0}(j)=\frac{(n+1-j)(n+2-j)}{2(n+1)(n+2)}, \\
& b_{0}(j)=\frac{j(-3+j-2 n)}{2(n+1)(n+2)}, \\
& b_{1}(j)=\frac{j\left(1+j+4 n+2 n^{2}\right)}{2(n+1)(n+2)} .
\end{aligned}
$$

However, the infinity-norm of its inverse was not presented in that paper. This is the first time constructive approach is used to model the properties of non-symmetric Teoplitz matrices.

In this paper, a set of band matrices of bandwidth $(r+s+1)$, where $s$ is the upper bandwidth and $r$ is the lower bandwidth are considered. The coefficients are derived from the binomial expansion $(x-1)^{r+s}$ and are placed about the diagonal in row and column fashion. The matrix of the type $A(2 r+1, n)$ where $2 r+1$ is the bandwidth has been studied by Hoskins and Ponzo [16], where the matrix is symmetric, for example

$$
A(5, n)=\left[\begin{array}{cccccc}
6 & -4 & 1 & & & \\
-4 & 6 & -4 & 1 & & \\
1 & -4 & 6 & -4 & 1 & \\
& & \ldots & \ldots & & \\
& 1 & -4 & 6 & -4 & 1 \\
& & 1 & -4 & 6 & -4 \\
& & & 1 & -4 & 6
\end{array}\right] .
$$

Herein, results for the determinants, inverses and infinity-norm of Non-symmetric, Odd and Even banded Toeplitz matrices arising from binomial coefficients are given. Also, constructive approach is used to derive results for different kinds of matrix classes of the type

$$
A(2 s \pm(t \pm 1), n)=(-1)^{s+j-i}\left(\begin{array}{c}
2 s \pm t \\
s+j-i
\end{array}\right) \text { as } t \longrightarrow \infty,
$$

where $s$ is the upper bandwidth of these matrices. Theorem 3 and Lemma 5 are similar to the results obtained by Arikan and Kilic [1] but with a novel approach. The matrix classes here are the same as [1] but the used method is different.

\section{Main Results}

For positive arbitrary integers $r, s$ and $t$ define the matrix $a_{i j}$ with upper bandwidth of $s$ and lower bandwidth of $r$ as

$$
a_{i j}=(-1)^{r+i-j}\left(\begin{array}{c}
2 r \mp t \\
r+i-j
\end{array}\right)=(-1)^{s+j-i}\left(\begin{array}{c}
2 s \pm t \\
s+j-i
\end{array}\right), \quad r, s \geq 0, t \in[0, \infty] .
$$

In the equation above, $r$ and $s$ can be arbitrarily chosen as positive integers. An example, which would be treated in this paper, is setting $r=s+1$. The inverse of the above matrix class is stated below.

\section{Theorem 1.}

The inverse of matrix $A(r+s+1, n)$ where $r+s+1$ represents the bandwidth is given by

$$
\begin{aligned}
\left\{A(r+s+1, n)^{-1}\right\}_{i, j} & =(-1)^{s}\left(\begin{array}{c}
i+s-1 \\
s
\end{array}\right)\left(\begin{array}{c}
j+r-1 \\
r
\end{array}\right) \\
& \times \sum_{k=i}^{n} \frac{\left(\begin{array}{c}
k+r-1-i \\
r-1
\end{array}\right)\left(\begin{array}{c}
k+s-1-j \\
s-1
\end{array}\right)}{\left(\begin{array}{c}
k+s-1 \\
s
\end{array}\right)\left(\begin{array}{c}
k+r+s-1 \\
r
\end{array}\right)} .
\end{aligned}
$$


The elements derived are those on the main diagonal and below. Other elements are obtained by symmetry, i.e., for values of $k$ where $j \leq k \leq n$. Related results of this are given by the following Corollaries.

\section{Corollary 1.}

Let $A[2 r \mp(t \mp 1), n]$ be a non-symmetric matrix of this class and $r$ be the lower bandwidth. If $L_{r}(i)=i(i+1)(i+$ 2) $\cdots(i+r-1)$, then, the elements on the diagonal and below are given by

$$
\begin{aligned}
\left\{A(2 r \mp(t \mp 1), n)^{-1}\right\}_{i, j} & = \pm \frac{(n+1-i)(n+2-i) \cdots(n+r \mp t-i)}{(r-1) !(2 r \mp(t \pm 1)) !} \\
& \times \sum_{v=0}^{r-1}\left[(-1)^{v}\left(\begin{array}{c}
r-1 \\
v
\end{array}\right) \frac{L_{r}(i)}{(i+v)} \frac{(j+v+r \mp(t \pm 1)) !}{(j+v-r) !} \frac{(n+v) !}{(n+v+r \mp t) !}\right], \quad i \geq j, \quad r \geq t \pm 1 .
\end{aligned}
$$

\section{Corollary 2.}

Let $A(2 s \pm(t \pm 1), n)$ be a non-symmetric matrix of this class and $s$ be the upper bandwidth. If $L_{s}(j)=j(j+$ 1) $\cdots(j+s+1)$, then, the elements on the diagonal and above are given by

$$
\begin{aligned}
\left\{A(2 s \pm(t \pm 1), n)^{-1}\right\}_{i, j} & =\mp \frac{(n+1-j)(n+2-j) \cdots(n+s \pm t-j)}{(s-1) !(2 s \pm(t \mp 1)) !} \\
& \times \sum_{k=0}^{s-1}\left[(-1)^{k}\left(\begin{array}{c}
s-1 \\
k
\end{array}\right)\left[\frac{L_{s}(j)}{j+k}\right] \frac{(j+k+s \pm(t \mp 1)) !}{(j+k-s) !} \frac{(n+k) !}{(n+k+s \pm t) !}\right], \quad i \leq j, \quad s \geq t \mp 1 .
\end{aligned}
$$

The Hoskins-Ponzo theorem (4) is easily derived when $s=r, v=k$ and $t=0$.

\subsection{The Bandwidth Case $2 s+2, \quad s \geq 1$ and $2 r-1, \quad r \geq 2$}

For non-negative values of $r$ and $s$, define the non-symmetric band matrices $A=\left\{a_{i j}\right\}$ in terms of binomial coefficients given by

$$
a_{i j}=(-1)^{r+j-i}\left(\begin{array}{c}
2 r-1 \\
r+j-i
\end{array}\right)=(-1)^{s+i-j}\left(\begin{array}{c}
2 s+1 \\
s+i-j
\end{array}\right) .
$$

For example, when $s=2, \quad r=3$ and $t=1$, the resulting matrix is

$$
A(6, n)=\left[\begin{array}{cccccccc}
10 & -5 & 1 & & & & & \\
-10 & 10 & -5 & 1 & & & & \\
5 & -10 & 10 & -5 & 1 & & & \\
-1 & 5 & -10 & 10 & -5 & 1 & & \\
& -1 & 5 & -10 & 10 & -5 & 1 & \\
& & & \cdots & \cdots & \cdots & & \\
& & -1 & 5 & -10 & 10 & -5 & 1 \\
& & & -1 & 5 & -10 & 10 & -5 \\
& & & & -1 & 5 & -10 & 10
\end{array}\right]
$$

The inverse of the matrix $A^{-1}$, is given by the theorems below. 


\section{Corollary 3.}

Let $A(r+s+1, n)$ be a non-symmetric matrix of this class and $r$ be the lower bandwidth. If $L_{r}(i)=i(i+1)(i+$ 2) $\cdots \cdots(i+r-1)$, then, the elements on the diagonal and below are given by

$$
\begin{aligned}
\left\{A(2 r, n)^{-1}\right\}_{i, j} & =\frac{(n+1-i)(n+2-i) \cdots(n+r-2-i)(n+r-1-i)}{(r-1) !(2 r-2) !} \\
& \times \sum_{k=0}^{r-1}\left[(-1)^{k}\left(\begin{array}{c}
r-1 \\
k
\end{array}\right) \frac{L_{r}(i)}{(i+k)} \frac{(j+k+r-2) !}{(j+k-r) !} \frac{(n+k) !}{(n+k+r-1) !}\right], \quad i \geq j, \quad r \geq 2 .
\end{aligned}
$$

The first three inverses for $i \geq j$ are given by

$$
\begin{aligned}
& \left\{A(4, n)^{-1}\right\}_{i, j}=\frac{(n+1-i)}{2(n+1)(n+2)}[(i+1) j(j-1)(n+2)-i(j+1) j(n+1)], \\
& \left\{A(6, n)^{-1}\right\}_{i, j}=\frac{(n+1-i)(n+2-i)}{48(n+1)(n+2)(n+3)(n+4)}[(i+1)(i+2)(j+1) j(j-1)(j-2)(n+4)(n+3) \\
& -2 i(i+2)(j+2)(j+1) j(j-1)(n+1)(n+4) \\
& +i(i+1)(j+3)(j+2)(j+1) j(n+2)(n+1)],
\end{aligned}
$$

and

$$
\begin{aligned}
& \left\{A(8, n)^{-1}\right\}_{i, j}=\frac{(n+1-i)(-i+n+2)(n+3-i)}{4320(n+1)(n+2)(n+3)(n+4)(n+5)(n+6)} \\
& \cdot[(i+1)(i+2)(i+3)(j+2)(j+1) j(j-1)(j-2)(j-3) \\
& -3 i(i+2)(i+3)(j+3)(j+2)(j+1) j(j-1)(j-2) \\
& +3 i(i+1)(i+3)(j+4)(j+3)(j+2)(j+1) j(j-1) \\
& -i(i+1)(i+2)(j+5)(j+4)(j+3)(j+2)(j+1) j] .
\end{aligned}
$$

\section{Corollary 4.}

Let $A(r+s+1, n)$ be a non-symmetric matrix of this class and s be the upper bandwidth. If $L_{s}(i)=j(j+1)(j+$ 2) $\cdots \cdots \cdots(j+s-1)$, then, the elements on the diagonal and above are given by

$$
\begin{aligned}
\left\{A^{-1}(2 s+2, n)\right\}_{i, j} & =\frac{(n+1-j)(n+2-j) \cdots(n+s-j)(n+s+1-j)}{(s-1) !(2 s) !} \\
& \times \sum_{k=0}^{s-1}\left[(-1)^{k}\left(\begin{array}{c}
s-1 \\
k
\end{array}\right) \frac{L_{s}(j)}{(j+k)} \frac{(i+k+s) !}{(i+k-s) !} \frac{(n+k) !}{(n+k+s+1) !}\right], \quad i \leq j, \quad s \geq 1 .
\end{aligned}
$$

The first three inverses for $i \leq j$ are given by

$$
\begin{aligned}
& \left\{A(4, n)^{-1}\right\}_{i, j}=\frac{(n+1-j)(n+2-j)(i+1) i}{2(n+1)(n+2)}, \\
& \left\{A(6, n)^{-1}\right\}_{i, j}=\frac{(n+1-j)(n+2-j)(n+3-j)}{24(n+1)(n+2)(n+3)(n+4)}[(j+1)(i+2)(i+1) i(i-1)(n+4) \\
& -j(i+3)(i+2)(i+1) i(n+1)], \\
& \left\{A(8, n)^{-1}\right\}_{i, j}=\frac{(n+1-j)(n+2-j)(n+3-j)(n+4-j)}{1440(n+1)(n+2)(n+3)(n+4)(n+5)(n+6)} \\
& \cdot[(j+1)(j+2)(i+3)(i+2)(i+1) i(i-1)(i-2)(n+5)(n+6) \\
& -2 j(j+2)(i+4)(i+3)(i+2)(i+1) i(i-1)(n+1)(n+6) \\
& +j(j+1)(i+5)(i+4)(i+3)(i+2)(i+1) i(n+1)(n+2)] .
\end{aligned}
$$

The determinant of the general Toeplitz matrix classes in Eq. (6) is defined in the theorems below 


\section{Theorem 2.}

For $r, s, n \geq 0$,

$$
\operatorname{det} A(r+s+1, n)=(-1)^{n+s-1} \prod_{k=1}^{n}\left(\begin{array}{c}
k+s+r-1 \\
r
\end{array}\right)\left(\begin{array}{c}
k+r-1 \\
r
\end{array}\right)^{-1}
$$

From the Lemma above, the determinants of the class of matrices (10) are given more elaborately. The first few terms are

$$
\begin{aligned}
\operatorname{det} A(4, n) & =\frac{(n+1)(n+2)}{1 \cdot 2} \\
\operatorname{det} A(6, n) & =\frac{(n+1)(n+2)^{2}(n+3)^{2}(n+4)}{1 \cdot 2^{2} \cdot 3^{2} \cdot 4} \\
\operatorname{det} A(8, n) & =\frac{(n+1)(n+2)^{2}(n+3)^{3}(n+4)^{3}(n+5)^{2}(n+6)}{1 \cdot 2^{2} \cdot 3^{3} \cdot 4^{3} \cdot 5^{2} \cdot 6} .
\end{aligned}
$$

Attention is then drawn to the Norm-infinity of general Toeplitz matrices' inverse arising from binomial coefficients by the following Corollary.

\section{Corollary 5.}

Let $r, s$ and $t$ be non-negative constants then

$$
\begin{aligned}
\left\|A(2 s \pm(t \pm 1), n)^{-1}\right\|_{\infty} & =\frac{\prod_{k=1}^{r}[n+2 k \pm(2 t \mp 1)] \prod_{m=1}^{s}[n+2 m \mp(2 t \pm 1)]}{2^{r+s}(r+s) !}, \text { for odd } \mathrm{n}, \\
& =\frac{\prod_{k=1}^{r}[n+2 k \pm(2 t \mp 2)] \prod_{m=1}^{s}[n+2 m \mp 2 t]}{2^{r+s}(r+s) !}, \text { for even } \mathrm{n} .
\end{aligned}
$$

When $t=0$ and $r=s$, Eqn. (3) is easily arrived at. The similar result is given below.

\section{Corollary 6.}

Norm-infinity of matrix class (10)'s inverses is stated as

$$
\begin{aligned}
\left\|A(2 s+2, n)^{-1}\right\|_{\infty} & =\frac{\prod_{k=1}^{r}(n+2 k+1) \prod_{m=1}^{s}(n+2 m-3)}{2^{r+s}(r+s) !}, \text { for odd } \mathrm{n}, \\
& =\frac{\prod_{k=1}^{r}(n+2 k) \prod_{m=1}^{s}(n+2 m-2)}{2^{r+s}(r+s) !}, \text { for even } \mathrm{n} .
\end{aligned}
$$

The first few terms for odd $n$ are given as

$$
\begin{aligned}
& \left\|A(4, n)^{-1}\right\|_{\infty}=\frac{1}{48}(n+3)(n+5)(n-1), \quad n \neq 1, \\
& \left\|A(6, n)^{-1}\right\|_{\infty}=\frac{1}{3840}(n+3)(n+5)(n+7)(n-1)(n+1), \quad n \neq 1, \\
& \left\|A(8, n)^{-1}\right\|_{\infty}=\frac{1}{645120}(n+3)^{2}(n+5)(n+7)(n+9)(n-1)(n+1), \quad n \neq 1 .
\end{aligned}
$$

Also, the first few terms for even $n$ are given as

$$
\begin{aligned}
& \left\|A(4, n)^{-1}\right\|_{\infty}=\frac{1}{48}(n+2)(n+4), \\
& \left\|A(6, n)^{-1}\right\|_{\infty}=\frac{1}{3840}(n+2)^{2}(n+4)(n+6) n, \\
& \left\|A(8, n)^{-1}\right\|_{\infty}=\frac{1}{645120}(n+2)^{2}(n+4)^{2}(n+6)(n+8) n .
\end{aligned}
$$

\section{Proofs}

In this section, Theorems 1, 2 and 3 are proved. 


\section{Proof of Theorem 1.}

Proof. For positive integers $j \geq 1$, consider

$$
\begin{aligned}
\eta_{j}(i) & =\frac{i(i+1)(i+2) \cdots(i+s-1)}{s !} \cdot \frac{(j+1-i)(j+2-i) \cdots(j+r-2-i)(j+r-1-i)}{(r-1) !} \\
& =\left(\begin{array}{c}
i+s-1 \\
s
\end{array}\right)\left(\begin{array}{c}
j+r-1-i \\
r-1
\end{array}\right) .
\end{aligned}
$$

Also consider, for any integer $i \geq 1$

$$
\begin{aligned}
\psi_{i}(j) & =\frac{j(j+1)(j+2) \cdots(j+r-1)}{r !} \cdot \frac{(i+1-j)(i+2-j) \cdots(i+s-2-j)(i+s-1-j)}{(s-1) !} \\
& =\left(\begin{array}{c}
j+r-1 \\
r
\end{array}\right)\left(\begin{array}{c}
i+s-1-j \\
s-1
\end{array}\right) .
\end{aligned}
$$

Then, $\eta_{j}(i)$ is a polynomial of degree $(r+s-1)$ in the variable $i$, where $\eta_{j}(i)=0$ for $i=0,-1,-2, \cdots,-s+1$ and $i=j+1, j+2, \cdots, j+r-1$. Also, $\psi_{i}(j)$ is a polynomial of degree $(r+s-1)$ in the variable $j$, where $\psi_{i}(j)=0$ for $j=0,-1,-2, \cdots,-r+1$ and $j=i+1, i+2, \cdots, i+s-1$. Give the definition of an $n$-vector $u_{j}$ where each component

$$
\begin{aligned}
u_{j}(i) & =\eta_{j}(i) \text { for } i<j+r \\
& =0 \text { for } i \geq j+r .
\end{aligned}
$$

Also, define the $n$-vector $l_{i}$ with components

$$
\begin{aligned}
l_{i}(j) & =\psi_{i}(j) \text { for } j<i+s \\
& =0 \text { for } j \geq i+s .
\end{aligned}
$$

Then $u_{j}(i)$ is the polynomial $\eta_{j}(i)$ [of degree $(r+s-1)$ ] for $i<j+r$, so that $\gamma^{r+s} u_{j}(i)=0$ for $i=1,2, \cdots, j-1$. Also, $l_{i}(j)$ is the polynomial $\psi_{i}(j)$ [of degree $(r+s-1)$ ] for $j<i+s$, so that $\delta^{r+s} l_{i}(j)=0$ for $j=1,2, \cdots, i-1$. If $U$ denotes the upper triangular matrix whose $j$ th column is $u_{j}$ as given in (19) and $L$ denotes the lower triangular matrix whose ith row is $l_{i}$, then $A U=L$. If $A(r+s+1, n)=\left\{a_{i j}\right\}$ where

$$
a_{i j}=(-1)^{r+i-j}\left(\begin{array}{c}
r+s \\
r+i-j
\end{array}\right) \text {, }
$$

and $U=\left\{u_{i j}\right\}$ where

$$
\begin{array}{rlr}
u_{i j} & =\left(\begin{array}{c}
i+s-1 \\
s
\end{array}\right)\left(\begin{array}{c}
j+r-1-i \\
r-1
\end{array}\right) & \text { for } i \leq j, \\
& =0 & \text { for } i>j,
\end{array}
$$

then

$$
A U=L .
$$

To continue the proof, the Theorem below is needed.

\section{Theorem 4:}

If the conditions of Theorem 1 are satisfied, then $A^{-1}=U D^{-1} U^{T}$, if the inverse exist.

Proof. From (21), $L=A U=\left(U^{T}\right)^{-1} D$. Hence, $A=\left(U^{T}\right)^{-1} D U^{-1}$ and $A^{-1}=U D^{-1} U^{T}$. Here, $T$ denotes the use of transposition. 
Continuing the proof, we determine $A(r+s+1, n)^{-1}$ by recalling from Theorem 4, that

$$
A(r+s+1, n)^{-1}=U D^{-1} U^{T} .
$$

Thus,

$$
\left\{A(r+s+1, n)^{-1}\right\}_{i, j}=\sum_{k=1}^{n} \frac{\eta_{i k} \psi_{k j}}{D_{k k}},
$$

where

$$
\begin{aligned}
& \eta_{i k}=\left(\begin{array}{c}
i+s-1 \\
s
\end{array}\right)\left(\begin{array}{c}
k+r-1-i \\
r-1
\end{array}\right), \\
& \psi_{k j}=\left(\begin{array}{c}
j+r-1 \\
r
\end{array}\right)\left(\begin{array}{c}
k+s-1-j \\
s-1
\end{array}\right) .
\end{aligned}
$$

Hence,

$$
\left\{A(r+s+1, n)^{-1}\right\}_{i, j}=(-1)^{s}\left(\begin{array}{c}
i+s-1 \\
s
\end{array}\right)\left(\begin{array}{c}
j+r-1 \\
r
\end{array}\right) \sum_{k=i}^{n} \frac{\left(\begin{array}{c}
k+r-1-i \\
r-1
\end{array}\right)\left(\begin{array}{c}
k+s-1-j \\
s-1
\end{array}\right)}{\left(\begin{array}{c}
k+s-1 \\
s
\end{array}\right)\left(\begin{array}{c}
k+r+s-1 \\
r
\end{array}\right)} .
$$

Remark:

When $r=s$, Eqn. (2) is easily derived.

Proof of Theorem 2.

Proof. From $A U=L$, the following is obtained

$$
\begin{aligned}
|A|=|L| /|U|=\prod_{k=1}^{n} l_{k k} / \prod_{k=1}^{n} u_{k k} & =\prod_{k=1}^{n}\left\{\frac{-\psi_{k}(k+s)}{\psi_{k}(k)}\right\} \\
& =\prod_{k=1}^{n}\left\{\frac{(-1)^{n}\left(\begin{array}{c}
k+s+r-1 \\
r
\end{array}\right)\left(\begin{array}{c}
-1 \\
s-1
\end{array}\right)}{\left(\begin{array}{c}
k+r-1 \\
r
\end{array}\right)}\right\} \\
& =(-1)^{n+s-1} \prod_{k=1}^{n} \frac{\left(\begin{array}{c}
k+s+r-1 \\
r
\end{array}\right)}{\left(\begin{array}{c}
k+r-1 \\
r
\end{array}\right)}
\end{aligned}
$$

where $l_{k k}=-\psi_{k}(k+s)$ and $u_{k k}=\gamma^{r+s} l_{k k}=\psi_{k}(k)$.

\section{Proof of Corollary 3.}

Proof. Before proving Corollary 3, the following Theorem is needed.

Theorem 5.

The row-sums, denoted by $S_{j}$ of $A^{-1}$ is given by

$$
S_{j}=(-1)^{S} \frac{\left(\begin{array}{c}
j+r-1 \\
r
\end{array}\right)\left(\begin{array}{c}
n-j+s \\
s
\end{array}\right)}{\left(\begin{array}{c}
r+s \\
s
\end{array}\right)}, \quad 1 \leq j \leq n
$$

Proof. By considering $\left(e_{j} U D^{-1} U^{T} e\right)$, for $j=1, \ldots, n$, given in Theorem 4, and, Theorem 3 in [16], the proof similarly follows. 


\section{Corollary 7.}

If $L$ and $U$ are the matrices of Theorem 1, then $L^{T} U=D$ a real diagonal matrix with

$$
D_{k k}=(-1)^{s}\left(\begin{array}{c}
k+s-1 \\
s
\end{array}\right)\left(\begin{array}{c}
k+s+r-1 \\
r
\end{array}\right)=(-1)^{s}\left(\begin{array}{c}
k+s+r-1 \\
r+s
\end{array}\right)\left(\begin{array}{c}
r+s \\
s
\end{array}\right) \text {. }
$$

Since $D=L^{T} U$, recall from Theorem 1 that $D_{k k}=l_{k k} u_{k k}$. From (19), $u_{k k}=\eta_{k}(k)$ and $l_{k k}=-\psi_{k}(k+s)$. Thus,

$$
u_{k k}=\eta_{k}(k)=\left(\begin{array}{c}
k+s-1 \\
r
\end{array}\right)\left(\begin{array}{c}
k+s-1-k \\
s-1
\end{array}\right)=\left(\begin{array}{c}
k+s-1 \\
s
\end{array}\right) \text {, }
$$

also

$$
\begin{aligned}
l_{k k}=\psi_{k}(k+s) & =(-1)\left(\begin{array}{c}
k+s+r-1 \\
r
\end{array}\right)\left(\begin{array}{c}
k+s-1-k-s \\
s-1
\end{array}\right) \\
& =(-1)\left(\begin{array}{c}
k+s+r-1 \\
r
\end{array}\right)\left(\begin{array}{c}
-1 \\
s-1
\end{array}\right) .
\end{aligned}
$$

Then

$$
D_{k k}=(-1)\left(\begin{array}{c}
k+s-1 \\
s
\end{array}\right)\left(\begin{array}{c}
k+s+r-1 \\
r
\end{array}\right)\left(\begin{array}{c}
-1 \\
s-1
\end{array}\right) \text {. }
$$

Also, note that

$$
\begin{gathered}
\left(\begin{array}{c}
-1 \\
s-1
\end{array}\right)=(-1)^{s-1} . \\
D_{k k}=(-1)^{s}\left(\begin{array}{c}
k+s+r-1 \\
r
\end{array}\right)\left(\begin{array}{c}
k+s-1 \\
s
\end{array}\right) .
\end{gathered}
$$

Moreover, simple calculations proceeding from (24) imply that

$$
D_{k k}=(-1)^{s}\left(\begin{array}{c}
k+s+r-1 \\
r+s
\end{array}\right)\left(\begin{array}{c}
r+s \\
s
\end{array}\right) \text {. }
$$

Acknowledgements: Effects of Professor S. N. Jator, Prof. S.K. Adegbie, Dr. Areo, Dr. A. I. Adekunle, Dr. S. Folarin, Dr. Dawodu, and other staff members of the Department of Mathematical Sciences, FUTA on the first author are highly appreciated. The first author also expresses his profound gratitude to the authorities of the Mathematics Laboratory for allowing him use the laboratory during the period of research.

Data Availability Statement: Data sharing is not applicable to this article as no datasets were generated or analyzed during the current study.

\section{References}

[1] T. Arikan and E. Kiliç, A class of non-symmetric band determinants with the Gaussianq-binomial coefficients, Quaestiones Mathematicae, vol. 40, no. 5, pp. 645-660, 2017.

[2] R. B. Bapat, An interlacing theorem for tridiagonal matrices, Linear Algebra and its Applications, vol. 150, pp. 331-340, 1991.

[3] W. Barrett, $A$ theorem on inverse of tridiagonal matrices, Linear Algebra and its Applications, vol. 27, pp. 211-217, 1979.

[4] J. H. Bevis and F. J. Hall, Integer LU-factorizations, Linear Algebra and its Applications, vol. 150, pp. 267-285, 1991.

[5] L. Brugnano and D. Trigiante, Tridiagonal matrices: Invertibility and conditioning, Linear Algebra and its Applications, vol. 166, pp. 131-150, 1992.

[6] D-qi Chen, An invariant deflation for lower banded matrices, Linear Algebra and its Applications, vol. 49, pp. $237-245,1983$.

[7] J. Dancis, "Invertible completions of band matrices," Linear Algebra and its Applications, vol. 150, pp. 125-138, 1991. 
[8] M. Dow, Explicit inverses of Toeplitz and associated matrices, ANZIAM Journal, vol. 44, p. 185, 2008.

[9] A. J. Durán, \& M. D. la Iglesia. Some examples of orthogonal matrix polynomials satisfying odd order differential equations. Journal of Approximation Theory, 150(2), 153-174, 2008.

[10] M. El-Mikkawy and A. Karawia, Inversion of general tridiagonal matrices, Applied Mathematics Letters, vol. 19, no. 8, pp. 712-720, 2006.

[11] B. Friedlander, M. Morf, T. Kailath, and L. Ljung, New inversion formulas for matrices classified in terms of their distance from Toeplitz matrices, Linear Algebra and its Applications, vol. 27, pp. 31-60, 1979.

[12] I. Gohberg, M. A. Kaashoek, and H. J. Woerdeman, A note on extensions of band matrices with maximal and submaximal invertible blocks, Linear Algebra and its Applications, vol. 150, pp. 157-166, 1991.

[13] T. N. E. Greville and W. F. Trench, Band matrices with Toeplitz inverses, Linear Algebra and its Applications, vol. 27, pp. 199-209, 1979.

[14] T. N. E. Greville, Toeplitz matrices with Toeplitz inverses revisited, Linear Algebra and its Applications, vol. 55, pp. 87-92, 1983.

[15] S. B. Haley, Solution of Band Matrix Equations by Projection-Recurrence, Linear Algebra and its Applications, vol. 32, pp. 33-48, 1980.

[16] W. D Hoskins and P. J. Ponzo, Some Properties of a Class of Band Matrices, Mathematics of computation, Volume 26, Number 118,1972

[17] C. R. Johnson and E. A. Schreiner, Explicit Jordan form for certain block triangular matrices, Linear Algebra and its Applications, vol. 150, pp. 297-314, 1991.

[18] F.-R. Lin, An explicit formula for the inverse of band triangular Toeplitz matrix, Linear Algebra and its Applications, vol. 428, no. 2-3, pp. 520-534, 2008.

[19] X.-G. Lv and T.-Z. Huang, A note on inversion of Toeplitz matrices, Applied Mathematics Letters, vol. 20, no. 12, pp. 1189-1193, 2007.

[20] D. S. Meek, The inverses of Toeplitz band matrices, Linear Algebra and its Applications, vol. 49, pp. 117-129, 1983.

[21] K. Murota, LU-decomposition of a matrix with entries of different kinds, Linear Algebra and its Applications, vol. 49, pp. 275-283, 1983.

[22] S. V. Parter, On the extreme eigenvalues of Toeplitz matrices, Transactions of the American Mathematical Society, vol. 100, no. 2, pp. 263-263, 1961.

[23] A. Pinkus, Matrices and $n$-Widths, Linear Algebra and its Applications, vol. 27, pp. 245-278, 1979.

[24] P. Rózsa, R. Bevilacqua, On band matrices and their inverses, Linear Algebra and its Applications, vol. 150, pp. 287-295, 1991.

[25] W. F. Trench, Characterization and properties of matrices with generalized symmetry or skew symmetry, Linear Algebra and its Applications, vol. 377, pp. 207-218, 2004.

[26] P. Van Dooren, The Computation of Kronecker's Canonical Form of a Singular Pencil, Linear Algebra and its Applications, vol. 32, pp. 103-14, 1979.

[27] C. F. Van Loan and J. P. Vokt, Approximating Matrices with Multiple Symmetries, SIAM Journal on Matrix Analysis and Applications, vol. 36, no. 3, pp. 974-993, 2015.

[28] K. Veselić, On real eigenvalues of real tridiagonal matrices, Linear Algebra and its Applications, vol. 27, pp. 167-171, 1979.

[29] J. F. Watters, Block Triangularization of Algebras of Matrices, Linear Algebra and its Applications, vol. 32, pp. 3-7, 1980.

[30] H.-J. Werner, On the matrix monotonicity of generalized inversion, Linear Algebra and its Applications, vol. 27, pp. 141-145, Oct. 1979.

[31] H. J. Werner, Some further results on matrix monotonicity, Linear Algebra and its Applications, vol. 150, pp. 371-392, 1991. 